\section{SEGREGACIÓN RESIDENCIAL SOCIOECONÓMICA EN LA CIUDAD DE CÓRDOBA (ARGENTINA): TENDENCIAS Y PATRONES ESPACIALES ${ }^{1}$}

Florencia Molinatti

\section{SOCIOECONOMIC RESIDENTIAL SEGREGATION IN CÓRDOBA (ARGENTINA): TRENDS AND SPATIAL PATTERNS ${ }^{1}$}

Florencia Molinatti²

\section{Resumen}

Desde hace algunos años se ha incrementado en América Latina el interés por el estudio de la segregación residencial socioeconómica, principalmente como consecuencia del notorio incremento de las desigualdades sociales y de los procesos de privatización que han tenido lugar en las ciudades de la región. Considerando que la mayoría de la investigación empírica ha tendido a documentar este fenómeno en las grandes metrópolis, este trabajo pretende contribuir a su estudio en ciudades intermedias. A partir de microdatos censales, se indaga cuán segregado socialmente está el espacio urbano de la ciudad de Córdoba (Argentina) en los años 1991, 2001 y 2008, en

\begin{abstract}
There has been a growing interest in the study of socioeconomic residential segregation over the last years in Latin America, mainly as a consequence of the evident increase in the social inequalities and privatization processes that have taken place in the cities of the region. Whereas most empirical research has tended to document this phenomenon in large cities, this paper aims to contribute to this field focusing the study in intermediate cities. Based on data retrieved from census microdata, the paper explores social segregation within the urban space of Cordoba as registered in 1991, 2001 and 2008 in terms of spatial concentration and social
\end{abstract}


términos de concentración espacial y homogeneidad social. Asimismo, mediante la aplicación de medidas de autocorrelación espacial, se analiza cómo se expresa dicha segregación en el espacio de la ciudad.

\section{PALABRAS CLAVE: SEgREGACIÓN - CIUDADES INTERMEDIAS - OCUPACIÓN DIFERENCIAL DEL ESPACIO}

Fecha de recepción: 02.03.13

Fecha de aceptación: 10.09.13

1 El presente artículo se estructura en base al aporte de las reflexiones y de los resultados obtenidos en la Tesis de Magíster de la autora. La misma fue financiada, en primer lugar, por el Consejo Nacional de Investigaciones Científicas y Técnicas (CONICET) a través del programa de becas doctorales y en segundo lugar, por el Proyecto de Investigación "Políticas habitacionales y segregación residencial socioeconómica en la ciudad de Córdoba", dirigido por el Dr. Enrique Peláez (CIECS-CONICET) y financiado por el Ministerio de Ciencia y Tecnología de la Provincia de Córdoba (Convocatoria PIO Sociales 2009), período 2010-2012.

2 Argentina. Licenciada en Sociología, Universidad Siglo 21, 2008. Magíster en Demografía, Universidad Nacional de Córdoba, 2013. Doctor(C) en Demografía, Universidad Nacional de Córdoba. Becaria Doctoral del Consejo Nacional de Investigaciones Científicas y Técnicas (CONICET). Correo electrónico: fmolinatti@hotmail.com. homogeneity. Furthermore, through the implementation of spatial autocorrelation measures, it is analyzed how such segregation is expressed in the urban space.

\section{KEYWORDS: SEGREGATION - INTERMEDIATE CITIES - DIFFERENTIAL OCCUPANCY OF SPACE}

Received: 02.03.13

Accepted: 10.09.13

1 This paper is based on the reflections and results of the master's thesis conducted by the author. This research was financed by the National Council for Scientific and Technological Development (CONICET) through the doctoral fellowship program and the research project "Housing policies and socioeconomic residential segregation in Córdoba", directed by Enrique Peláez, PhD (CIECS-CONICET) and financed by the Ministry of Science and Technology, Córdoba (PIO Socials Program 2009), 2010-2012.

2 Argentina. BA in Sociology, Siglo 21 University, 2008. MSc in Demography, National University of Córdoba, 2013. Doctoral student, National University of Cordoba. Doctoral fellow, National Council for Scientific and Technological Development (CONICET).Email: fmolinatti@hotmail.com. 


\section{Introducción}

El acelerado proceso de urbanización que caracterizó a los países de América Latina, por lo menos hasta finales de la década de 1970, tuvo como uno de sus resultados mega-ciudades con una alta desigualdad por ingresos, hogares en viviendas de condición deficitaria y acceso desigual a bienes y servicios públicos. Sin embargo, en las últimas décadas se registraron una serie de cambios que provocaron rupturas en las tendencias observadas. Unos de los más relevantes se refieren al retroceso de la primacía urbana, a la desaceleración del crecimiento de las mega-ciudades y al crecimiento de las ciudades de tamaños intermedios y la multiplicación de su número 3 .

En gran medida, los estudios orientados a documentar los patrones y tendencias de la localización diferencial de los grupos sociales en el espacio urbano latinoamericano se han centrado casi exclusivamente en las mega-ciudades. Aunque, se destacan algunas investigaciones recientes $^{4}$ que ilustran la importante presencia y relevancia que la segregación residencial y los procesos a ella asociados tienen en aquellas de menor tamaño.

3 Cunha y Jiménez, 2009; Lattes, 2001; Vapñarsky, 1995.

4 Cunha y Jiménez, 2009; Gómez, 2011; Linares, 2012; Marengo, 2004; Peláez, González y Cunha, 2008; Prieto, Formiga y Medus, 2011; Sabatini, Cáceres y Cerda, 2001; Tecco y Valdés, 2006; entre otros.
Este trabajo se orienta a analizar cuantitativamente las tendencias de la segregación residencial socioeconómica (SRS) en la ciudad de Córdoba ${ }^{5}$ (República Argentina) en los años 1991 y 2001 y 2008, y a determinar cómo se expresa esa segregación en el espacio urbano. Esta ciudad constituye el segundo aglomerado urbano del país, con una población de 1.329.604 habitantes, según los datos del último Censo Nacional de 2010. Desde comienzos de 1980, la ciudad viene experimentando dos tipos de transformaciones en su estructura: por un lado, una desaceleración de las tasas de crecimiento de la población y, por otro lado, un conjunto de transformaciones cualitativas en las modalidad de estructuración del territorio, manifiestas en los nuevos patrones de localización de las actividades residenciales y comerciales ${ }^{6}$.

La primera de estas transformaciones significó un retroceso en el acelerado ritmo de crecimiento poblacional registrado en el siglo XX y, especialmente, entre 1940 y 1960 asociado al proceso de industrialización. Este retroceso se evidenció en tasas de crecimiento medias anuales cada vez menores, aunque todavía positivas: en el último período inter-censal (2001-10) se registró un crecimiento medio anual de 3,8 cada 1000 habitantes, siendo este

5 La ciudad de Córdoba, capital de la provincia homónima, se localiza a 703 kilómetros al Noroeste de la Ciudad Autónoma de Buenos Aires (ciudad capital de la República Argentina).

$6 \quad$ Municipalidad de Córdoba, 2008. 
valor un $55 \%$ y un $75 \%$ menor que los registrados entre 1991-2001 y 1980-1991, respectivamente.

Con relación a las transformaciones cualitativas en la estructura urbana, Marengo ${ }^{7}$ manifiesta que la ciudad de Córdoba se encuentra experimentado, desde finales de los años ochenta del siglo XX, un proceso de expansión suburbana, que se produce conjuntamente con la reestructuración del espacio metropolitano, la concentración económica y la localización selectiva de nuevas inversiones privadas, fundamentalmente en la periferia urbana. Este proceso se evidencia en la cantidad y en la diversidad de las intervenciones habitacionales desarrollados en los bordes de la ciudad.

Por un lado, la urbanización privada se encuentra principalmente asociada al crecimiento del parque residencial de la ciudad a partir del diseño y la comercialización de emprendimientos bajo la modalidad de urbanizaciones de perímetro cerrado conocidas como countries con equipamientos diversos (club-houses, canchas de tenis, campos de golf, centros de servicios), condominios verticales de lujo, entre otros. Según datos de la Municipalidad de Córdoba ${ }^{8}$,

7 Marengo, 2002.

8 Municipalidad de Córdoba, 2008. entre 1985 y 2008 se incorporaron 42 countries y barrios cerrados, representando casi el 10\% de las nuevas urbanizaciones registradas. Por otro lado, y como contracara de este proceso, el Estado (provincial y municipal) ha desarrollado urbanizaciones destinadas a la localización/relocalización de población de bajos ingresos en la periferia de la ciudad, siendo la más relevante, al menos en términos cuantitativos, el Programa "Mi Casa, Mi Vida" ejecutado entre 2003 y 2007 por el Gobierno de la Provincia ${ }^{9}$. El total de las intervenciones estatales representan casi el 17\% de las 447 nuevas urbanizaciones tramitadas entre 1985 y $2008^{10}$.

Asimismo, en esta ciudad, como en la mayoría de las ciudades latinoamericanas, el acceso al suelo de una gran parte de la población se encuentra ligado con la necesidad y con la participación en acciones colectivas. Esta lógica de acceso se expresa en una serie de tipologías de loteos caracterizadas, principalmente, por la autoproducción colectiva y por la irregularidad/informalidad. Por diversos motivos resulta muy difícil establecer cuantificaciones sobre la escala de este fenómeno;

9 Estas relocalizaciones fueron realizadas en el marco del Programa "Mi Casa, Mi Vida", a partir del cual se trasladaron alrededor de 7.000 familias desde asentamientos informales localizados en zonas próximas al área central o barrios pericentrales hacia grandes complejos habitacionales construidos en la periferia semi-rural de la ciudad.

10 Ibíd. 
aunque, estimaciones ofrecidas desde la Dirección de Hábitat de la Municipalidad de Córdoba indican que esa cifra no superaría el 20\% de las viviendas existentes ${ }^{11}$.

En este contexto, el proceso de expansión urbana hacia la periferia y la consiguiente distribución geográfica de las nuevas urbanizaciones en la ciudad de Córdoba ofrecerían indicios sobre los procesos de producción y apropiación del espacio, determinados en gran medida por los diferentes patrones de movilidad residencial que se dan en el interior de la ciudad. En este artículo se pretende aportar evidencias, a partir del análisis de la segregación residencial socioeconómica, acerca de estos indicios que sobresalen del estudio de los procesos de urbanización que han tenido lugar en la ciudad de Córdoba desde las últimas dos décadas del siglo XX.

A estos fines, este trabajo se estructura en cinco partes, además de la presente introducción. Primero, se presentan sucintamente algunos conceptos y lineamientos teorías que atraviesan a la noción de SRS. Segundo, se explicita qué se entiende por SRS y qué dimensiones objetivas presenta. Tercero, se detalla las fuentes de información y los métodos utilizados en este trabajo. Cuarto, se exhiben los resultados alcanzados. Por último, se discuten dichos resultados a

11 Rebord, 2010. la luz de las transformaciones socio-urbanísticas recientes y se presentan las principales conclusiones arribadas.

\section{Hacia una conceptualización de la segregación residencial socioeconómica}

\section{LOS MÚLTIPLES SENTIDOS DE LA PALABRA SEGREGACIÓN}

Brun $^{12}$, quien ha hecho numerosas contribuciones epistemológicas sobre el significado del concepto segregación, argumenta que esta noción no es lo suficiente precisa como para ser utilizada como una herramienta conceptual rigurosa. La polisemia y la ambigüedad que caracteriza este concepto, debido a la fuerte orientación semántica que ha cargado la palabra desde sus orígenes, invitan a los investigadores y estudiosos del tema a contextualizar el uso de este término. En todos los casos, el sentido es dado, en gran parte, por el tipo de aproximación que se quiera hacer del tema. Desde luego, no es un concepto unívoco, el término tiene diversas dimensiones y utilizaciones.

12 Brun, 1994. 
En su sentido etimológico, la segregación, de acuerdo a la definición de la Real Academia Española, resulta de la acción de separar o de apartar algo de otra u otras cosas. Sin embargo, esta idea puede ser entendida de diversas formas. Desde el punto de vista sociológico, la palabra puede significar la ausencia de interacción entre grupos sociales; en cambio, cuando es avocada en un sentido geográfico, segregación puede referirse a la desigualdad en la distribución de los grupos sociales a través del espacio físico. La presencia de un tipo de segregación no implica necesariamente la presencia de la otra. Por ejemplo, en la sociedad de castas la segregación sociológica puede ser casi absoluta, aún cuando los miembros de las diferentes castas estén próximos en el espacio físico. No obstante, es probable que ambos tipos de segregación estén correlacionadas ${ }^{13}$.

De manera general, algunos autores ${ }^{14}$ coinciden en considerar que el sentido más tradicional de la palabra segregación remite a la imagen del ghetto, donde una fracción de la población es relegada dentro de los espacios habitacionales estrictamente circunscriptos a causa de su condición étnica o racial. Se ha utilizado entonces para designar los ghettos judíos, también para describir los problemas raciales entre grupos

13 White, 1983, p. 1009

14 Brun y Rhein, 1994; Marcuse, 2001, Grafmeyer, 1994. étnicos dentro de la sociedad norteamericana y dentro del sistema del apartheid en Sudáfrica. Como consecuencia de estos usos, en muchas ocasiones la palabra es asociada directamente con un sentido de discriminación. Sin embargo, con el tiempo el contenido de la palabra ha evolucionado y ha adquirido nuevos sentidos, en algunos casos más o menos ambiguos, a medida que comienza a designar nuevos fenómenos ${ }^{15}$.

Grafmeyer ${ }^{16}$ considera que si se circunscribe a la segregación a su dimensión residencial pueden encontrarse dos abordajes interdependientes, pero conceptualmente distintos, de dicha noción:

- El primero se refiere a la identificación de las diferencias de localización entre grupos definidos en función de criterios tales como la posición social, el origen étnico, la religión, etc. Un grupo de población se considera segregado cuando se encuentra distribuido de forma desigual entre las diferentes zonas de una ciudad. En este sentido, la segregación residencial es producto de las lógicas individuales, teniendo como fin el resguardo frente a los efectos fragmentadores de la personalidad generados por la aglomeración

15 Salas Vanegas, 2008.

16 Grafmeyer, 1994. 
y vida en la ciudad. Este abordaje presenta una larga tradición en los estudios raciales y étnicos realizados en los Estados Unidos, en especial aquellos vinculados con la concepción ecológica de la Escuela de Chicago.

- El segundo pone acento sobre las oportunidades desiguales de acceso a los bienes materiales y simbólicos ofrecidos por la ciudad. De esta forma, la segregación residencial es resultado de las luchas entre los grupos por la apropiación de los recursos materializados en la ciudad en el marco de la sociedad capitalista. Este abordaje es muy bien ilustrado por los trabajos que el Centro de Sociología Urbana en Francia consagró a la cuestión de los equipamientos colectivos entre las décadas de 1970 y 1980.

Ambas concepciones significaron importantes contribuciones al estudio de la segregación residencial, aunque en diferentes sentidos. Mientras los principales aportes de la visión ecologista de la relación espacio-sociedad se encuentran en el desarrollo de diversos índices y medidas para la cuantificación del fenómeno y en el desarrollo de diferentes modelos de evolución urbana (por ejemplo, el modelo de anillos concéntricos de Burgess), la incorporación de la noción de distribución desigual significó una desnaturalización del fenómeno

ARTÍCULO: Segregación residencial socioeconómica en la ciudad de Córdoba (Argentina) Tendencias y patrones espaciales / Florencia Molinatti y una explicitación de cómo los grupos o clases sociales luchan por apropiarse de la ciudad como un recurso.

En ese último sentido, y a pesar que la segmentación social tiene manifestaciones territoriales, la estructura espacial no es un mero reflejo de la estructura social, ya que como expresa Bourdieu ${ }^{17}$ "... el espacio social se retraduce en el espacio físico, pero siempre de manera más o menos turbia; el poder sobre el espacio que da la posesión del capital en sus diversas especies se manifiesta en el espacio físico apropiado en la forma de determinada relación entre la estructura espacial de las distribución de los agentes y la estructura espacial de la distribución de los bienes o servicios, privados o públicos".

La segregación residencial, entonces, corresponde a una forma espacial y arquitectónica de la ciudad que se retroalimenta de un tipo de interacción social que contribuye poco a la conformación de relaciones sociales más inclusivas, justas y democráticas. Según un estudio realizado por el Programa de Naciones Unidas para el Desarrollo (PNUD) para los cuatro aglomerados urbanos principales de Argentina, la segregación urbana propicia la naturalización y la profundización de las diferentes y contribuye así a aumentar la vulnerabilidad de los grupos de menores recursos ${ }^{18}$.

17 Bourdieu, 1999, p. 120

18 PNUD, 2009.

revista invi № 79 / Noviembre 2013 / Volumen № 28: 61-94 
Como lo han demostrado numerosos trabajos, la segregación residencial tiene importantes efectos sociales negativos, concretos y medibles, especialmente sobre los grupos de menores recursos y vulnerables. No solo es consecuencia de la pobreza, sino que es también un poderoso determinante de su reproducción. Por un lado, afecta a los hogares más pobres en su desempeño educativo ${ }^{19}$. Por otro lado, incide negativamente en la inserción laboral y en la relación con el mercado de trabajo ${ }^{20}$. Ambos fenómenos reducen las probabilidades de movilidad social y restringen las oportunidades productivas.

Otros autore ${ }^{21}$ explicitan que la segregación también produce un proceso de enclaustramiento excluyente de los ricos, quienes, junto con su autosegregación en zonas residenciales exclusivas, tienden a concentrar bienes y servicios en su entorno, atenuando los requerimientos de circular por la ciudad y las posibilidades de interactuar con personas de menor nivel socioeconómico fuera de su entorno inmediato.

19 Alves, Franco y Ribeiro, 2008; Groisman y Suárez, 2006; Kaztman y Retamoso, 2007; Molinatti y Peláez, 2012; PNUD, 2009.

20 Cunha y Jakob, 2010; Kaztman \& Retamoso, 2005; Molinatti, 2013; PNUD, 2009

21 Caldeira, 2007; Svampa, 2004.

\section{DELIMITACIÓN DE LA NOCIÓN DE SEGREGACIÓN RESIDENCIAL SOCIOECONÓMICA}

La segregación residencial es un fenómeno espacial con complejas y contradictorias relaciones con las diferencias y desigualdades sociales, las cuales suelen causar importantes confusiones con relación a qué se entiende por segregación y qué dimensiones distinguibles presenta ${ }^{22}$.

La segregación residencial remite a la existencia de desigualdades en la distribución de las localizaciones cotidianas de las personas o sus familias que residen en un determinado aglomerado urbano ${ }^{23}$. En esta línea, la segregación residencial puede definirse, en términos generales, como "... el grado de proximidad espacial $o$ de aglomeración territorial de las familias pertenecientes a un mismo grupo social, sea que este se defina en términos étnicos, etarios, de preferencias religiosas o socioeconómicas" ${ }^{24}$. En términos más específicos, la segregación residencial ${ }^{25}$ presenta dos dimensiones objetivas: (1) la tendencia

\footnotetext{
22 Rodríguez y Arriagada, 2004; Sabatini, 2003.

23 Rodríguez Vignoli, 2001; Sabatini et al., 2001.

24 Sabatini, Cáceres y Cerda 2001, p. 27.

25 La SRS también presenta una tercera dimensión, de carácter subjetivo. Se refiere representaciones que los miembros de un grupo social tienen de los lugares (zonas o barrios) que ellos mismos habitan y de los lugares que habitan personas pertenecientes a otros grupos sociales. Esta dimensión debería estudiarse preferentemente con metodologías cualitativas, quedando fuera del análisis propuesto en este trabajo.
} 
de los grupos sociales a concentrarse en algunas áreas de la ciudad; y (2) la conformación de áreas o barrios con un alto grado de homogeneidad social ${ }^{26}$.

Estas dimensiones parecen, a primera vista, dos formas de mirar lo mismo; sin embargo, cada una capta un aspecto distinto del fenómeno. La distinción se encuentra en que mientras la primera analiza los grupos sociales en términos de su localización espacial (concentración/dispersión), la segunda examina las áreas en términos de su composición social (homogeneidad/ heterogeneidad) ${ }^{27}$.

Con relación a los atributos que actúan como diferenciadores de los grupos poblacionales, se observa que gran parte de las investigaciones en América Latina analizan la segregación residencial de acuerdo a diferentes variables de estratificación socioeconómica, tales como nivel educativo, ingresos, categoría socio-ocupacional, condiciones materiales de vida, entre otras ${ }^{28}$. De acuerdo con Sabatini ${ }^{29}$ “... es un ángulo comprensible considerando que las fuertes desigualdades sociales, de ingreso y de rango o

6 Sabatini, 2003; Sabatini, Cáceres y Cerda, 2001.

Sabatini, Cáceres y Cerda, 2001

Rodríguez Vignoli, 2001; Sabatini, 2003.

Sabatini, 2003, p.13. clase social, representan tal vez la característica más saliente de la estructura social de los paises de América Latina -más que la pobreza, en todo caso". En este trabajo, se exploran los procesos de SRS, utilizando a la vivienda como punto de referencia para localizar espacialmente a los grupos sociales y a sus atributos socioeconómicos como criterio diferenciador.

\section{Metodología}

\section{FUENTES DE DATOS Y VARIABLE UTILIZADAS}

Las fuentes de datos utilizadas en esta investigación fueron secundarias y el análisis de la SRS se realizó a partir de información recolectada en tres Censos de Población: los dos primeros (1991 y 2001) tuvieron cobertura nacional y fueron relevados por el Instituto Nacional de Estadística y Censos (INDEC), y, el último (2008), con una cobertura a nivel provincial, estuvo a cargo de la Dirección General de Estadísticas y Censos de la Provincia de Córdoba $(\mathrm{DGEC})^{30}$.

30 En octubre de 2010 se llevó a cabo el último Censo Nacional de Población; sin embargo, sus resultados no pudieron ser incorporados en este trabajo porque, al momento de su elaboración, no se encontraban disponibles al público los microdatos censales. Según información provista por el INDEC, los mismos serían entregados en formato REDATAM a finales del 2013.

revista invi № 79 / Noviembre 2013 / Volumen № 28: 61-94 
Respecto a la escala geográfica de agregación utilizada, el criterio de división territorial a utilizarse se encuentra condicionado por la disponibilidad de datos residenciales de la población. Actualmente, la información censal se encuentra desagregada, para los tres años considerados, a nivel de fracción, radio y segmento censal. A los fines operacionales, el INDEC realiza tres tipos de subdivisiones censales (fracciones, radios y segmentos). En orden descendente, el Departamento Capital -donde se localiza la ciudad de Córdoba- se divide en fracciones, las fracciones en radios y los radios en segmentos ${ }^{31}$. Sólo la información a nivel de fracción y radio censal se encuentra disponibles para los usuarios, ya que la agregada a nivel de segmento se encuentra resguardada por la Ley de Secreto Estadístico (Ley ${ }^{\circ}$ 17.622/68).

Debido a la ausencia de datos referidos a los ingresos de los individuos y de los hogares a nivel censal, se propuso cuantificar la SRS para cada uno de los años y analizar su evolución a partir de un indicador proxy del nivel socioeconómico del

31 La cantidad de fracciones y radios censales pueden variar entre censo y censo, principalmente como consecuencia de los cambios en los tamaños y densidades poblacionales. Por ejemplo, en Córdoba la cantidad de fracciones se mantuvo constante en 84 unidades, pero la cantidad de radios aumentó de 1.126 a 1.377 entre 1991 y 2008, siendo 1.375 en el año 2001. hogar, el máximo nivel educativo del jefe del hogar ${ }^{32}$ categorizado en:

- Bajo: Sin estudios o con primario incompleto

- Medio-bajo: Con primario completo o secundario incompleto.

- Medio-alto: Con secundario completo o superior incompleto.

\section{- Alto: Con superior completo}

Diversos autores ${ }^{33}$ argumentan que esta variable se caracteriza por tener un gran poder de segmentación en las ciudades latinoamericanas, principalmente por la creciente significación del conocimiento en las nuevas formas de producción y, por ende, en la determinación de los diferenciales en los salarios y en las protecciones sociales a las que tienen acceso los trabajadores con distintos niveles de calificación. Asimismo, la elección de esta variable se fundamenta en su posibilidad de comparabilidad entre los diferentes Censos; para lo cual, se compatibilizaron sus categorías, procurando lograr la mayor similitud entre ellas. Cabe destacar que las preguntas referidas a la educación fueron realizadas a todos los jefes de hogar que residían al momento de los tres censos en la ciudad de

32 Para evitar el efecto distorsionador de la estructura etaria sobre la educación de una población y procurando captar a un grupo de particular influencia se trabajó sólo con los jefes y las jefas de hogar de 30 a 59 años.

33 Kaztman \& Retamoso, 2005; Rodríguez, 2008; Sabatini, et. al, 2009 
Córdoba, siendo los valores incluidos en este trabajo poblacionales.

\section{MÉTODOS}

Como una primera aproximación a la medición de la SRS se optó por una serie de medidas tradicionales ampliamente utilizadas para cuantificar la segregación no sólo de las ciudades de América Latina sino de todas partes del mundo. Estas medidas fueron seleccionadas también por su pertinencia para proporcionar una información sintética y global respecto a las dos dimensiones objetivas del fenómeno: la concentración espacial y la homogeneidad social.

Los dos primeros indicadores (Índice de Disimilitud de Duncan (D) ${ }^{34}$ e Índice de Segregación Residencial (IS) ${ }^{35}$ ) hacen alusión a la igualdad en la

34

$$
\mathrm{D}=\frac{1}{2} \sum\left|\frac{N_{1 j}}{N_{1}}-\frac{N_{2 i}}{N_{2}}\right| ; 0 \leq D \leq 1
$$

Donde $\mathrm{D}$ es el índice de disimilitud de Duncan; $\mathrm{N} 1 \mathrm{i}$ = población del grupo 1 en subdivisión territorial i-ésima; N2i = población del grupo 2 en subdivisión territorial i-ésima; N1 = población del grupo 1 en la unidad territorial superior; $y$ N2 = población del grupo 2 en la unidad territorial superior.

IS $=\frac{1}{2} \sum\left|\frac{N_{1 j}}{N_{1}}-\frac{N_{i}-N_{1 j}}{N-N_{1}}\right| ; 0 \leq I S \leq 1 ;$

Donde IS es el índice de segregación; $\mathrm{N} 1 \mathrm{i}=$ población del grupo 1 en subdivisión territorial $\mathrm{i}$-ésima; $\mathrm{N} 1$ = población del grupo 1 en la unidad territorial superior; $\mathrm{Ni}$ = población total en subdivisión territorial i-ésima; y $\mathrm{N}$ = población total en la unidad territorial superior. distribución de uno o más grupos en las zonas o en las unidades espaciales en que podemos dividir un espacio geográfico y toman como referencia los parámetros del conjunto de la ciudad. Ambos varían entre 0 (ausencia de segregación) y 1 (segregación total), y, multiplicados por cien, pueden ser interpretados como la proporción de habitantes que debería modificar su lugar de residencia con el fin de obtener en cada área una representación idéntica a la proporción del grupo en el conjunto urbano. La diferencia entre ellos consiste en que mientras que el IS mide la distribución de un grupo respecto del total de la población de la ciudad, el D compara las proporciones de dos grupos entre $\mathrm{si}^{36}$.

El tercer indicador (Índice de Aislamiento Corregido $\left.\left(\eta^{2}\right)^{37}\right)$ indica el grado de homogeneidad social de las áreas de residencia. Se dice que un grupo está

\footnotetext{
36 Massey y Denton, 1988.

37 $\eta^{2}=\frac{{ }_{x} P_{x}-P}{1-P} ;_{x} P_{x}=\sum_{i=1}^{n}\left(\frac{x_{i}}{x}\right) *\left(\frac{x_{i}}{t_{i}}\right)$

Donde $\eta^{2}$ es el índice de aislamiento corregido; $P_{x}$ es índice de aislamiento sin corregir; $P$ es la proporción de población del grupo $X$ en la ciudad; $x_{i}$ es la población del grupo $x$ en la subdivisión territorial i-ésima; $X$ es la población del grupo $x$ en la ciudad; $y t$ es la población total en la subdivisión territorial i-ésima.
} 
segregado si presenta un aislamiento fuerte, es decir, que la mayoría de sus contactos potenciales se darían con miembros de su mismo grupo. Este índice varía entre 0 (ausencia de segregación) y 1 (segregación total) $^{38}$.

En la lectura e interpretación de los índices de segregación es importante tener en cuenta el rol de la escala de análisis en el valor del índice -este efecto se conoce en la literatura especializada como el "problema del tablero de ajedrez"-. La diferente evaluación de una misma situación objetiva obedece sólo al nivel de agregación escogido. Por ejemplo, si una zona de la ciudad estuviera habitada sólo por miembros de una clase social sería evidentemente segregada. Sin embargo, si se observa dentro de la zona la idea de segregación carecería de sentido, ya que todos sus residentes pertenecen a la misma clase social ${ }^{39}$.

$\mathrm{Al}$ ser un problema intrínsecamente metodológico, diversos autores ${ }^{40}$ aconsejan como tratamiento la utilización de las mismas escalas geográficas de medición cuando se estudia distintas ciudades o la misma en diferentes momentos. De acuerdo con estos autores, mientras mayor sea el tamaño del área de medición,

38 Debido a que el índice clásico de aislamiento es extremadamente sensible a la representación proporcional de los diferentes grupos -es decir, el índice presenta mayores valores en aquellas poblaciones con mayor número de casos-, se trabajó con la versión corregida de este índice $\left(\eta^{2}\right)$.

39 Rodríguez Vignoli, 2001.

40 Caprón y González Arrelano, 2006; Reardon y 0'Sullivan, 2004; Sabatini et al., 2009.

72 revista invi № 79 / Noviembre 2013 / Volumen № 28: 61-94 menor será el índice, acercándose a cero cuando esta área tiende a igualarse con el área total de la ciudad; y cuanto menor sea el área de medición, mayor será el valor del índice, tendiendo al valor de uno al acercarse al tamaño mínimo posible, el del hogar.

A fin de contemplar este efecto, se incluyeron dos estrategias en el análisis de la SRS: por un lado, los datos censales fueron procesados para las dos desagregaciones disponibles (fracciones y radios censales) y, por otro lado, en el análisis gráfico de los valores del IS y las escalas en las cuales fue medido se aplicó una corrección metodológica, representada gráficamente por un recta diagonal, que se refiere a la disminución esperable del índice cuando aumenta el tamaño del área de medición. Esta corrección permitió distinguir entre el efecto esperable ante el cambio de escala y el efecto de la segregación propiamente dicho. Un curva por encima por encima de la recta indicaría que la escala de la segregación es alta (macro-segregación) y una curva por debajo, que la escala es baja (micro-segregación). El tamaño del área contenida entre la curva y la recta indica el grado en que la segregación varía en esa ciudad entre las distintas escalas geográficas ${ }^{41}$.

41 Sabatini et al., 2009 
Estas tres medidas resumen permitieron estimar y conocer el grado de segregación de la ciudad como un todo, pero tienen como limitación común que no posibilitan la identificación de las áreas segregadas en el espacio geográfico de la ciudad. Para esto último se necesita la utilización de medidas georreferenciadas, que tengan como punto de partida la semejanza (o no) de las unidades espaciales vecinas. En este trabajo se optó por una medida basada en la noción de autocorrelación espacial: el Índice de Moran, cuyos valores fueron ajustados, a fin de aislar el efecto del tamaño de la población de referencia, a partir de una Estandarización Empírica Bayesiana ${ }^{42}$.

El Índice de Moran es un indicador de asociación espacial que evalúa la existencia de conglomerados (clusters) en la distribución espacial de una determinada variable, pudiéndose ser calculado como medida general (Global) ${ }^{43}$ o de forma localizada

42 Assunçao y Reis, 1999.

43

$$
\mathrm{I}=\frac{n \sum_{i} \sum_{j} w_{i j}\left(x_{i}-\bar{x}\right)\left(x_{j}-\bar{x}\right)}{s_{o} \sum_{i}\left(x_{i}-\bar{x}\right)^{2}} ; \mathrm{s}_{o}=\sum_{i} \sum_{j>i} w_{i j}
$$

Donde I es el Índice de Moran Global; W es la matriz ponderada tal que wij es igual a 1 si las unidades espaciales son adyacentes 0 igual a cero en el caso contrario; $n$ es el número de unidades geográficas; xi es la proporción de población del grupo x en la subdivisión territorial i-ésima; xj es la proporción de población del grupo $x$ en la subdivisión territorial j-ésima; $\bar{x}$ es la proporción de población del grupo $x$ en la unidad territorial superior; $y$ so es la cantidad total de límites espaciales comunes en el área. para cada unidad espacial (Local) ${ }^{44}$. Mientras la versión global del índice ofrece un estadístico que permite establecer el grado de aglomeración de unidades semejantes, la versión local posibilita la creación de un mapa, en el que se pueden detectar las unidades espaciales que se encuentran rodeadas por otras semejantes o no ${ }^{45}$.

Los índices globales de segregación fueron obtenidos en una aplicación C++ desarrollada el Laboratoire d'analyse spatiale et d'économie régionale (LASER) disponible en http://laser.ucs.inrs. ca/EN/Download.html. En cambio, el cálculo del Índice de Moran en sus versiones global y local y la creación de los mapas de autocorrelación local fueron realizados en la versión 9.8.14 del software libre OpenGeoDa del GeoDa Center for Geospatial Analysis and Computation, disponible en https://geodacenter.asu.edu/software/ downloads.

44

$$
\mathrm{I}_{i}=\frac{\left(x_{i}-\bar{x}\right) \sum_{j} w_{i j}\left(x_{j}-\bar{x}\right)}{\sum_{i} \frac{\left(x_{i}-\bar{x}\right)^{2}}{N}}
$$

Donde li es el Índice Moran Local; $x_{i}$ la proporción de población del grupo $x$ en la subdivisión territorial i-ésima; $x_{i}$ la proporción de población del grupo $x$ en la subdivisión territorial j-ésima; $\bar{x}$ es la proporción de población del grupo $\mathrm{x}$ en la unidad territorial superior; $y w_{i j}$ es igual a 1 si las unidades espaciales son adyacentes o igual a 0 en el caso contrario. 
TABLA 1: VARIACIONES ABSOLUTAS Y RELATIVAS DEL MÁXIMO NIVEL EDUCATIVO DEL JEFE DE HOGAR. CIUDAD DE CÓRDOBA. AÑOS 1991, 2001 Y 2008.

\begin{tabular}{|c|c|c|c|c|c|c|c|c|c|}
\hline \multirow[t]{2}{*}{ Nivel educativo del Jefe de Hogar } & \multirow[t]{2}{*}{1991} & \multirow[t]{2}{*}{2001} & \multirow[t]{2}{*}{2008} & \multicolumn{2}{|c|}{$\begin{array}{l}\text { Variación } \\
\text { 1991-2001 }\end{array}$} & \multicolumn{2}{|c|}{$\begin{array}{l}\text { Variación } \\
\text { 2001-2008 }\end{array}$} & \multicolumn{2}{|c|}{$\begin{array}{l}\text { Variación } \\
\text { 1991-2008 }\end{array}$} \\
\hline & & & & $\Delta$ & $\Delta \%$ & $\Delta$ & $\Delta \%$ & $\Delta$ & $\Delta \%$ \\
\hline BAJO & 28.747 & 23.284 & 20.787 & & & & & & \\
\hline $\begin{array}{l}\text { Sin estudios o con primario } \\
\text { incompleto }\end{array}$ & $16 \%$ & $11 \%$ & $9 \%$ & -5.463 & $-19,0 \%$ & -2.497 & $-10,7 \%$ & -7.960 & $-27,7 \%$ \\
\hline MEDIO-BAJO & 84.936 & 84.800 & 80.574 & & & & & & \\
\hline $\begin{array}{l}\text { Con primario completo o con se- } \\
\text { cundario incompleto }\end{array}$ & $47 \%$ & $41 \%$ & $36 \%$ & -136 & $-0,2 \%$ & -4.226 & $-5,0 \%$ & -4.362 & $-5,1 \%$ \\
\hline MEDIO-ALTO & 42.045 & 61.190 & 72.683 & & & & & & \\
\hline $\begin{array}{l}\text { Con secundario completo o supe- } \\
\text { rior incompleto }\end{array}$ & $24 \%$ & $30 \%$ & $32 \%$ & 19.145 & $45,5 \%$ & 11.493 & $18,8 \%$ & 30.638 & $72,9 \%$ \\
\hline ALTO & 23.456 & 36.464 & 49.149 & & & & & & \\
\hline Con superior completo & $13 \%$ & $18 \%$ & $22 \%$ & 13.008 & $55,5 \%$ & 12.685 & $34,8 \%$ & 25.693 & $109,5 \%$ \\
\hline
\end{tabular}

Nota: el alcance territorial de los tres censos analizados corresponde a la totalidad del ejido municipal.

Fuente: Elaboración propia con base en información de los Censos 1991, 2001 y 2008.

\section{Resultados}

Antes de proceder con el análisis de la SRS propiamente dicha, se presentan las variaciones absolutas y relativas referidas a la variable de análisis, posibilitando identificar en qué contexto general se dan los cambios en los patrones residenciales. En términos generales, se observa una mejora de la situación socioeconómica de la población de la ciudad de Córdoba entre 1991 y 2008, en este

74 revista invi № 79 / Noviembre 2013 / Volumen N² 28: 61-94 trabajo caracterizada a partir del nivel educativo de los jefes de hogar que al momento de los censos tenían entre 30 y 59 años. Todas las categorías inferiores al secundario completo disminuyen su representación tanto en términos relativos como absolutos, incrementándose correlativamente el peso de las categorías superiores (Tabla 1). Esta evidente mejora en el nivel educativo es un fenómeno que puede responder a distintos factores, y no se debe entender mecánicamente como una movilidad 
TABLA 2: ÍNDICE DE SEGREGACIÓN RESIDENCIAL SEGÚN EL NIVEL EDUCATIVO DEL JEFE DE HOGAR Y LA ESCALA DE AGREGACIÓN. CIUDAD DE CÓRDOBA. AÑOS 1991, 2001 Y 2008.

\begin{tabular}{|c|c|c|c|c|}
\hline \multirow[t]{2}{*}{ Nivel educativo del Jefe de Hogar } & \multirow[t]{2}{*}{ Escala } & \multicolumn{3}{|c|}{ Año Censal } \\
\hline & & 1991 & 2001 & 2008 \\
\hline \multirow{2}{*}{ BAJO Sin estudios o con primario incompleto } & Radio & 39,3 & 42,9 & 44,9 \\
\hline & Fracción & 29,2 & 31,8 & 30,0 \\
\hline \multirow{2}{*}{ MEDIO-BAJO Con primario completo o con secundario incompleto } & Radio & 24,6 & 31,8 & 37,8 \\
\hline & Fracción & 18,7 & 25,7 & 31,0 \\
\hline \multirow{2}{*}{ MEDIO-ALTO Con secundario completo o superior incompleto } & Radio & 28,6 & 25,3 & 22,5 \\
\hline & Fracción & 21,5 & 18,2 & 15,7 \\
\hline \multirow[t]{2}{*}{ ALTO Con superior completo } & Radio & 47,4 & 46,8 & 46,0 \\
\hline & Fracción & 38,2 & 38,4 & 38,4 \\
\hline
\end{tabular}

Notas:

Porcentaje de población del grupo que debe cambiar su lugar de residencia. 100\% = máxima segregación.

El alcance territorial de los tres censos analizados corresponde a la totalidad del ejido municipal.

Fuente: elaboración propia con base en información de los Censos 1991, 2001 y 2008.

social ascendente de jefes que mejoraron su nivel de instrucción. Antes bien, es probable que se explique por otros factores tales como el recambio generacional a partir de la constitución de nuevos hogares con jefes más jóvenes y mejor instruidos que sus padres y abuelos.

En este contexto surge como interrogante cómo estos avances registrados en el campo educativo se han visto reflejados, junto con otros procesos socio-urbanísticos, en la ocupación diferencial del espacio urbano por parte de los grupos sociales que lo conforman.

\section{NIVELES DE SEGREGACIÓN RESIDENCIAL SOCIOECONÓMICA EN LA CIUDAD DE CÓRDOBA}

\section{GRADO DE CONCENTRACIÓN ESPACIAL DE LOS GRUPOS SOCIALES}

El IS muestra, para los tres años censales, que los jefes con alto nivel educativo son los que se encuentran más segregados, o menos homogéneamente distribuidos en el espacio geográfico de la ciudad, y los jefes con nivel educativo medio, los menos segregados. Por su parte, los jefes educación baja se encuentran en una situación intermedia; 
FIGURA 1: COMPARACIÓN DE LOS IS CON LA RECTA METODOLÓGICA SEGÚN EL NIVEL EDUCATIVO DEL JEFE DE HOGAR Y LA ESCALA DE AGREGACIÓN. CIUDAD DE CÓRDOBA, AÑOS 1991, 2001 Y 2008.

Fuente: elaboración propia con base en información de los Censos 1991, 2001 y 2008.

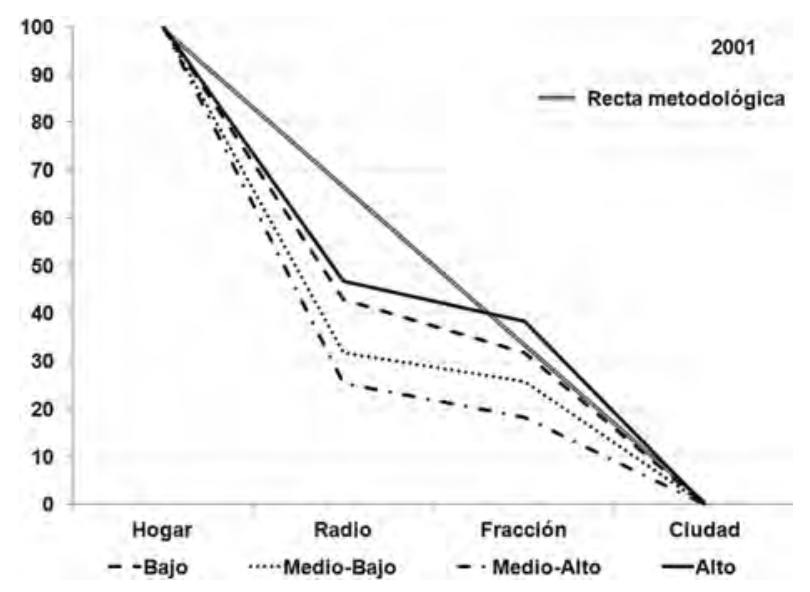

76 revista invi № 79 / Noviembre 2013 / Volumen № 28: 61-94
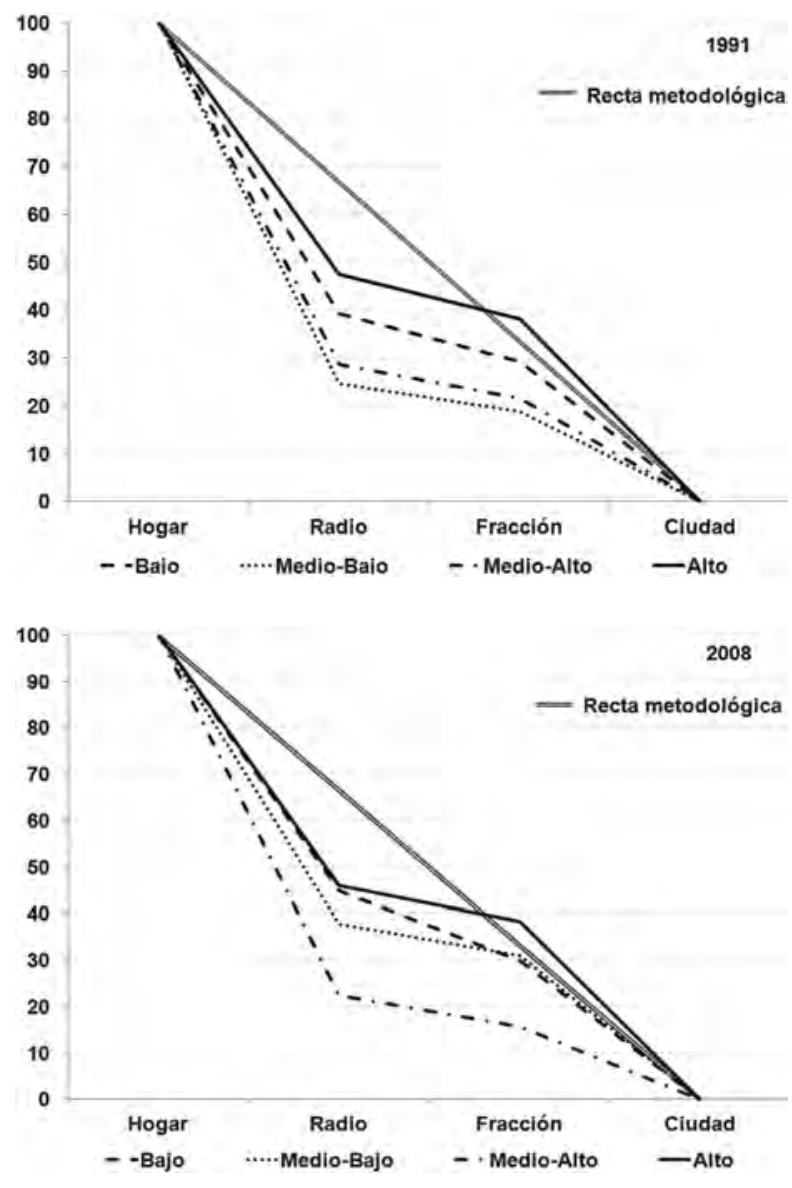
TABLA 3: ÍNDICE DE DISIMILITUD DE DUNCAN SEGÚN EL NIVEL EDUCATIVO DEL JEFE DE HOGAR Y LA ESCALA DE AGREGACIÓN. CIUDAD DE CÓRDOBA, AÑOS 1991, 2001 Y 2008.

\begin{tabular}{|c|c|c|c|c|c|c|}
\hline \multicolumn{2}{|c|}{ Nivel educativo del Jefe de Hogar } & & \multirow[t]{2}{*}{ Escala } & \multicolumn{2}{|c|}{ Año Censal } & \multirow[b]{2}{*}{2008} \\
\hline Grupo 1 & Grupo 2 & Relación 1-2 & & 1991 & 2001 & \\
\hline \multirow{2}{*}{ BAJO } & \multirow{2}{*}{ MEDIO-BAJO } & \multirow{2}{*}{ Contiguos } & Radio & 27,5 & 26,9 & 30,3 \\
\hline & & & Fracción & 18,4 & 16,3 & 14,0 \\
\hline \multirow{2}{*}{ MEDIO-BAJO } & \multirow{2}{*}{ MEDIO-ALTO } & \multirow{2}{*}{ Contiguos } & Radio & 33,1 & 34,9 & 36,8 \\
\hline & & & Fracción & 26,0 & 27,1 & 29,2 \\
\hline \multirow{2}{*}{ MEDIO-ALTO } & \multirow{2}{*}{ ALTO } & \multirow{2}{*}{ Contiguos } & Radio & 25,9 & 28,1 & 29,1 \\
\hline & & & Fracción & 19,6 & 21,5 & 22,7 \\
\hline \multirow{2}{*}{ BAJO } & \multirow{2}{*}{ MEDIO-ALTO } & \multirow{2}{*}{ Salteados } & Radio & 53,8 & 54,7 & 54,1 \\
\hline & & & Fracción & 40,4 & 41,0 & 37,5 \\
\hline \multirow{2}{*}{ MEDIO-BAJO } & \multirow{2}{*}{ ALTO } & \multirow{2}{*}{ Salteados } & Radio & 53,6 & 56,7 & 59,2 \\
\hline & & & Fracción & 42,9 & 46,6 & 49,7 \\
\hline \multirow{2}{*}{ ALTO } & \multirow{2}{*}{ BAJO } & \multirow{2}{*}{ Extremos } & Radio & 67,8 & 70,8 & 69,1 \\
\hline & & & Fracción & 55,5 & 57,3 & 54,3 \\
\hline
\end{tabular}

Notas:

Porcentaje de población del grupo que debe cambiar su lugar de residencia. 100\% = máxima segregación.

El alcance territorial de los tres censos analizados corresponde a la totalidad del ejido municipal.

Fuente: elaboración propia con base en información de los Censos 1991, 2001 y 2008.

aunque con valores más cercanos a los calculados para los jefes con alto nivel educativo. Dicho patrón parece no tener diferencias significativas según las escalas geográficas de medición y se asemeja al encontrado en otras ciudades latinoamericanas (Tabla 2).

Entre los cambios en los niveles de segregación observados para cada uno de los grupos sociales considerados se destacan:
1) El grupo de jefes de hogar con alto nivel educativo se mantuvo como el grupo más segregado de la ciudad en los tres años censales. En 2008 casi el 50\% de los hogares cuyos jefes tienen estudios superiores completos deberían cambiar su residencia para alcanzar una distribución más homogénea en la ciudad.

2) El grupo de jefes con educación baja se mantuvo en los años analizados como el segundo 
grupo más segregado. Debido al aumento del valor del IS en ambos períodos, su nivel de segregación se aproximó al observado para los jefes de mayor nivel educativo.

3) Los jefes con nivel educativo medio-alto conforman el único grupo que presentó retrocesos en los niveles de segregación en ambos períodos, constituyendo hacia 2008 el grupo menos segregado.

Sin embargo, considerando el efecto metodológico que la escala geográfica tiene sobre los valores que asume el índice de segregación, se procedió a comparar los valores del índice para cada grupo según las escalas de medición y a aplicar una corrección metodológica representada por una recta diagonal, que se refiere a la disminución esperable del índice cuando aumenta el tamaño del área de medición. En la Figura 1 se observa que las curvas de segregación de los diferentes grupos educativos se encuentran por debajo de la recta, indicado que la ciudad de Córdoba se caracteriza por ser una ciudad con segregación a baja escala (o migro-segregación). Esto significa que, más allá del efecto metodológico, los jefes de hogar según nivel educativo se encuentran más fuertemente segregados a menor escala. Asimismo se observa que entre 1991 y 2008 disminuyó el grado en que la segregación varía entre las diferentes escalas para los distintos grupos, representado por la disminución del área que se ubica entre la recta y las curvas de segregación.
El otro índice de concentración utilizado, D, muestra una estrecha correlación entre nivel educativo del jefe de hogar y la ocupación del espacio, sugiriendo una alta correspondencia entre la distribución espacial de los grupos y las distancias educativas existentes entre ellos. El índice alcanza valores más bajos al calcularse entre grupos educativos contiguos y valores más altos entre grupos salteados y extremos, especialmente entre estos últimos. En términos generales, el proceso de segregación por concentración espacial medida de a pares en el período considerado exhibe una tendencia hacia el aumento, reafirmándose la ocupación diferencial del espacio urbano (Tabla 3).

\section{GRADO DE HOMOGENEIDAD SOCIAL EN LAS ÁREAS INTERNAS DE LA CIUDAD}

Los resultados indican que todos grupos de jefes se encuentran relativamente poco segregados, ya que el grado de aislamiento obtenido para cada uno de ellos es inferior al 25\% en los tres años censales considerados. Esto indica que más de tres cuartas partes de sus potenciales contactos los realizarían con miembros de otros grupos. A pesar de estos bajos niveles de segregación, se destaca que el grupo más aislado es el grupo conformado por los jefes de hogar con mayor nivel educativo y el menos aislado aquel con educación media-alta (Tabla 4).

Entre los cambios que tuvieron lugar se destacan, por un lado, el aumento del aislamiento/segregación 
TABLA 4: ÍNDICE DE AISLAMIENTO CORREGIDO (П2), SEGÚN EL NIVEL EDUCATIVO DEL JEFE DE HOGAR Y LA ESCALA DE AGREGACIÓN. CIUDAD DE CÓRDOBA. AÑNOS 1991, 2001 Y 2008

\begin{tabular}{|c|c|c|c|c|}
\hline \multirow[t]{2}{*}{ Nivel educativo del Jefe de Hogar } & \multirow[t]{2}{*}{ Escala } & \multicolumn{3}{|c|}{ Año Censal } \\
\hline & & 1991 & 2001 & 2008 \\
\hline \multirow[t]{2}{*}{ BAJO Sin estudios o con primario incompleto } & Radio & 12,7 & 11,6 & 11,4 \\
\hline & Fracción & 6,6 & 5,6 & 4,2 \\
\hline \multirow[t]{2}{*}{ MEDIO-BAJO Con primario completo o con secundario incompleto } & Radio & 9,0 & 13,4 & 17,4 \\
\hline & Fracción & 5,6 & 9,1 & 11,8 \\
\hline \multirow[t]{2}{*}{ MEDIO-ALTO Con secundario completo o superior incompleto } & Radio & 8,2 & 7,5 & 6,5 \\
\hline & Fracción & 4,6 & 3,8 & 3,0 \\
\hline \multirow[t]{2}{*}{ ALTO Con superior completo } & Radio & 15,9 & 19,3 & 20,6 \\
\hline & Fracción & 10,4 & 13,2 & 14,3 \\
\hline
\end{tabular}

Notas:

Grado de exposición del grupo x al contacto con miembros del mismo grupo. 100\% = máxima segregación.

El alcance territorial de los tres censos analizados corresponde a la totalidad del ejido municipal.

Fuente: elaboración propia con base en información de los Censos 1991, 2001 y 2008.

de los jefes con educación alta y media-baja, y, por otro lado, el retroceso, aunque en menor proporción, del aislamiento/segregación de aquellos con educación baja y media-alta. Respecto a la magnitud de las variaciones, el mayor aumento tuvo lugar en el grupo de jefes con educación media-baja, tanto a nivel de radio como de fracción censal, acercándose en 2008 a los valores registrados para el grupo más segregado, jefes con educación alta.

ARTíCULO: Segregación residencial socioeconómica en la ciudad de Córdoba (Argentina): Tendencias y patrones espaciales / Florencia Molinatti

\section{IDENTIFICACIÓN DE LAS ÁREAS DE SEGREGACIÓN RESIDENCIAL SOCIOECONÓMICA ${ }^{46}$}

\section{GRADO DE AUTOCORRELACIÓN ESPACIAL}

Para el caso de la ciudad de Córdoba, el Índice de Moran Global presenta valores positivos y significativos de autocorrelación espacial para los cuatro grupos

46 Considerando que los hogares en la ciudad de Córdoba, en función de la variable de segmentación socioeconómica seleccionada, se encuentran mayormente segregados a baja escala, el análisis de autocorrelación espacial fue realizado sólo para la escala de agregación menor, es decir, a nivel de radio censal.

revista invi № 79 / Noviembre 2013 / Volumen $N^{\circ} 28: 61-94$ 


\section{TABLA 5: ÍNDICE DE MORAN GLOBAL SEGÚN EL NIVEL EDUCATIVO DEL JEFE DE HOGAR Y EL TIPO DE CONTIGÜIDAD, A NIVEL DE RADIO CENSAL. CIUDAD DE CÓRDOBA. AÑOS 1991, 2001 Y 2008.}

\begin{tabular}{lllllll} 
Nivel educativo del Jefe de Hogar & \multicolumn{3}{l}{ Contigüidad Queen $1^{\circ}$ Orden } & \multicolumn{4}{c}{ Contigüidad Rook $1^{\circ}$ Orden } \\
& \multicolumn{2}{c}{ Año Censal } & \multicolumn{4}{c}{ Año Censal } \\
& 1991 & 2001 & 2008 & 1991 & 2001 & 2008 \\
\hline BAJO Sin estudios o con primario incompleto & 0,6706 & 0,6401 & 0,4300 & 0,6708 & 0,6436 & 0,4330 \\
\hline $\begin{array}{l}\text { MEDIO-BAJO Con primario completo o con secundario } \\
\text { incompleto }\end{array}$ & 0,7267 & 0,7678 & 0,7848 & 0,7277 & 0,7675 & 0,7841 \\
\hline $\begin{array}{l}\text { MEDIO-ALTO Con secundario completo o superior } \\
\text { incompleto }\end{array}$ & 0,6827 & 0,6292 & 0,5943 & 0,6862 & 0,6320 & 0,5935 \\
\hline ALTO Con superior completo & 0,7714 & 0,7769 & 0,7936 & 0,7704 & 0,7772 & 0,7950 \\
\hline
\end{tabular}

Nota: valores p-seudo significativos al 0,001. Para todos los casos se rechaza hipótesis nula de aleatoriedad.

Fuente: elaboración propia con base en información de los Censos 1991, 2001 y 2008.

educativos, a partir del porcentaje de cada uno de ellos en los radios censales, independientemente del tipo de contigüidad utilizado (Queen y Rook con criterio de primer orden $)^{47}$. Esto significa que los jefes de hogar, según su máximo nivel educativo alcanzado, exhiben un patrón de distribución no aleatorio y, considerando el signo positivo de los índices, concentrado (Tabla 5).

Específicamente, los jefes de hogar con educación alta presentan un patrón de distribución espacial altamente concentrado. Este patrón no sólo se mantiene

47 Se espera que las diferentes entre las contigüidades Queen y Rook sean mínimas en áreas irregulares, como lo son los radios y las fracciones censales. Sin embargo, la literatura especializada recomienda evaluar la sensibilidad de los resultados cuando se cambia el tipo de contigüidad. en los tres años analizados, sino que el índice presenta un leve aumento entre 1991 y 2008, cercano al 3\% en su valor. Este grupo es seguido por el constituido por jefes con educación media-baja, quienes también presentan un patrón altamente concentrado y con un incremento del 8\% en el valor del índice en el período analizado. Los dos grupos restantes, a pesar de presentar valores significativos y positivos de autocorrelación espacial, exhiben un retroceso en su concentración espacial, siendo más pronunciado en el caso de los jefes con menor instrucción (tabla 5). 
FIGURA 2: MAPAS DE AUTOCORRELACIÓN ESPACIAL SEGÚN PORCENTAJE DE JEFES DE HOGAR CON EDUCACIÓN BAJA, A NIVEL DE RADIO CENSAL. CIUDAD DE CÓRDOBA, AÑOS 1991, 2001 Y 2008.
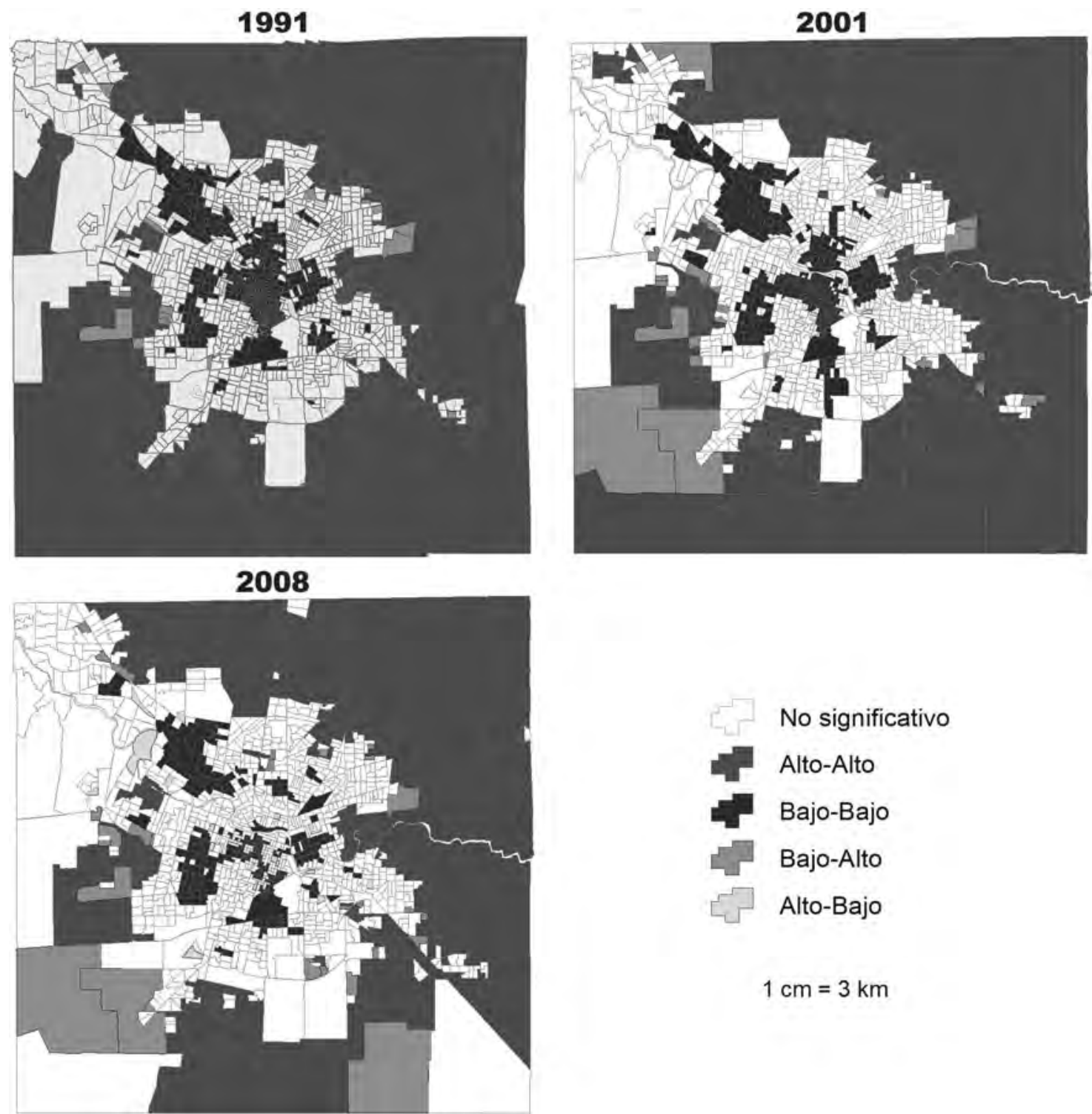

Fuente: elaboración propia con base en información de los Censos 1991, 2001 y 2008 y cartografía oficial de INDEC. 
FIGURA 3: MAPAS DE AUTOCORRELACIÓN ESPACIAL SEGÚN PORCENTAJE DE JEFES DE HOGAR CON EDUCACIÓN MEDIA-BAJA, A NIVEL DE RADIO CENSAL. CIUDAD DE CÓRDOBA, AÑOS 1991, 2001 Y 2008.
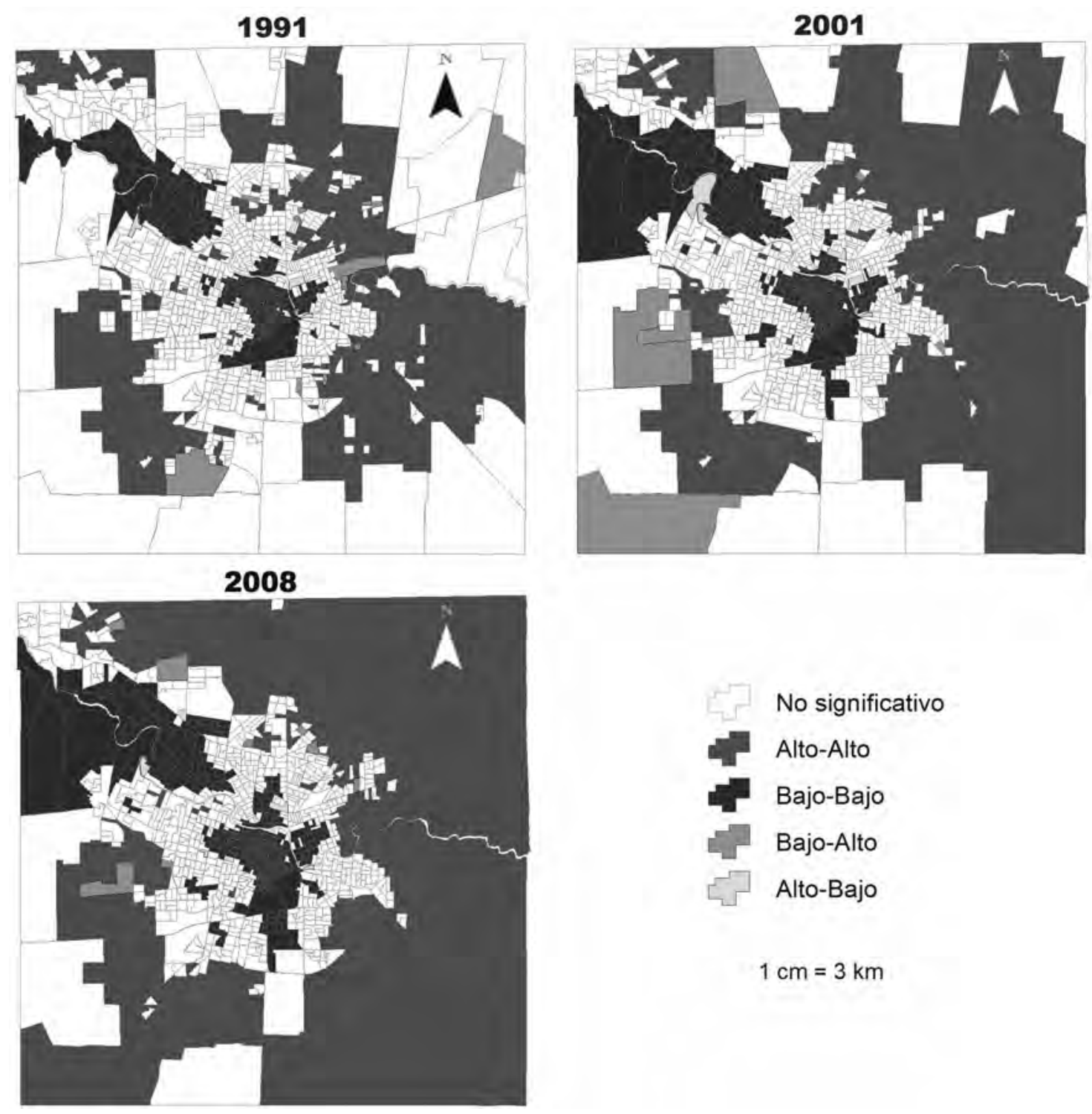

$1 \mathrm{~cm}=3 \mathrm{~km}$

Fuente: elaboración propia con base en información de los Censos 1991, 2001 y 2008 y cartografía oficial de INDEC. 
FIGURA 4: MAPAS DE AUTOCORRELACIÓN ESPACIAL SEGÚN PORCENTAJE DE JEFES DE HOGAR CON EDUCACIÓN MEDIA-ALTA, A NIVEL DE RADIO CENSAL. CIUDAD DE CÓRDOBA, AÑOS 1991, 2001 Y 2008.
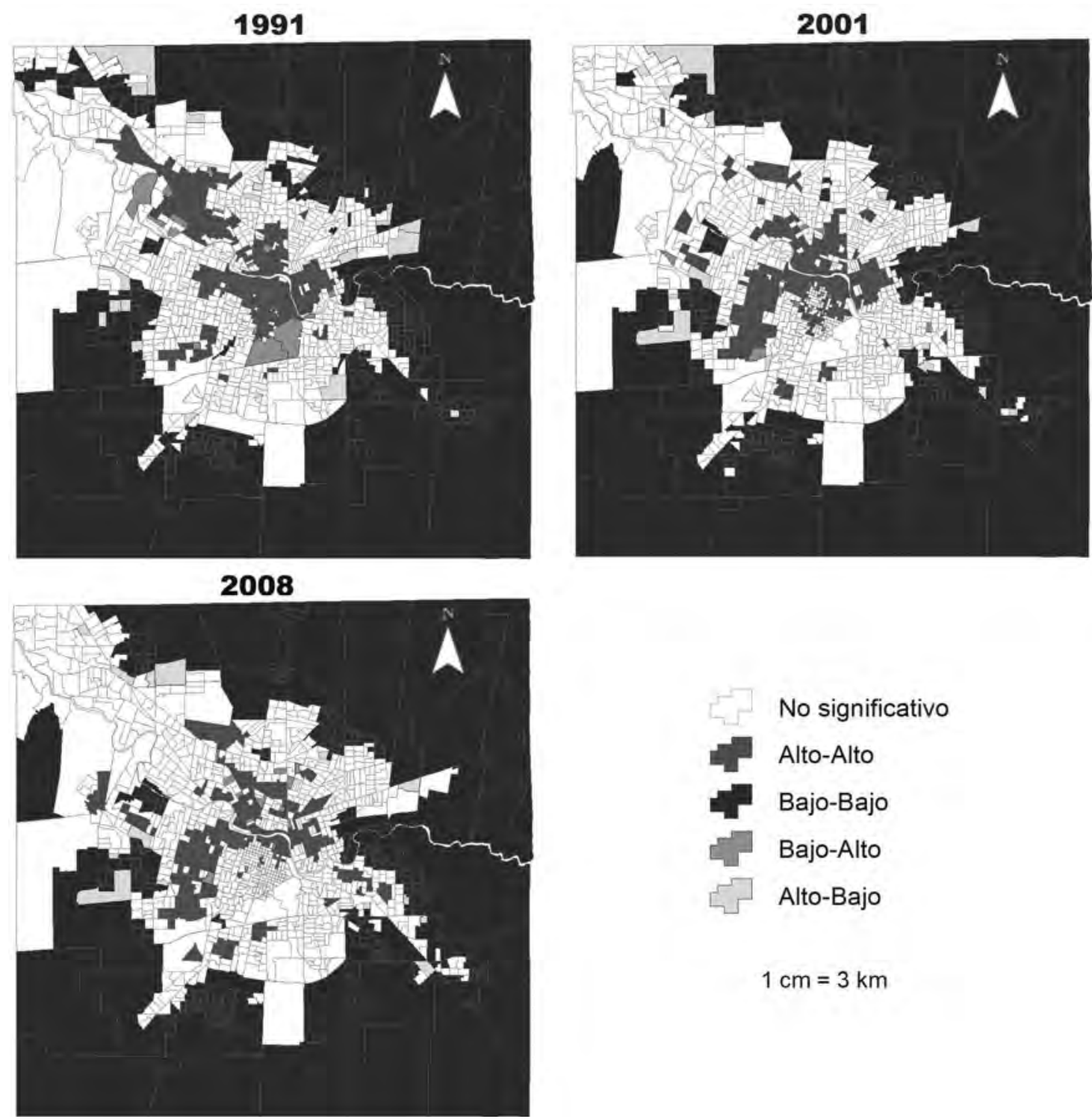

No significativo

Alto-Alto

Bajo-Bajo

Bajo-Alto

Alto-Bajo

$1 \mathrm{~cm}=3 \mathrm{~km}$

Fuente: elaboración propia con base en información de los Censos 1991, 2001 y 2008 y cartografía oficial de INDEC. 
FIGURA 5: MAPAS DE AUTOCORRELACIÓN ESPACIAL SEGÚN PORCENTAJE DE JEFES DE HOGAR CON EDUCACIÓN ALTA, A NIVEL DE RADIO CENSAL. CIUDAD DE CÓRDOBA, AÑOS 1991, 2001 Y 2008.
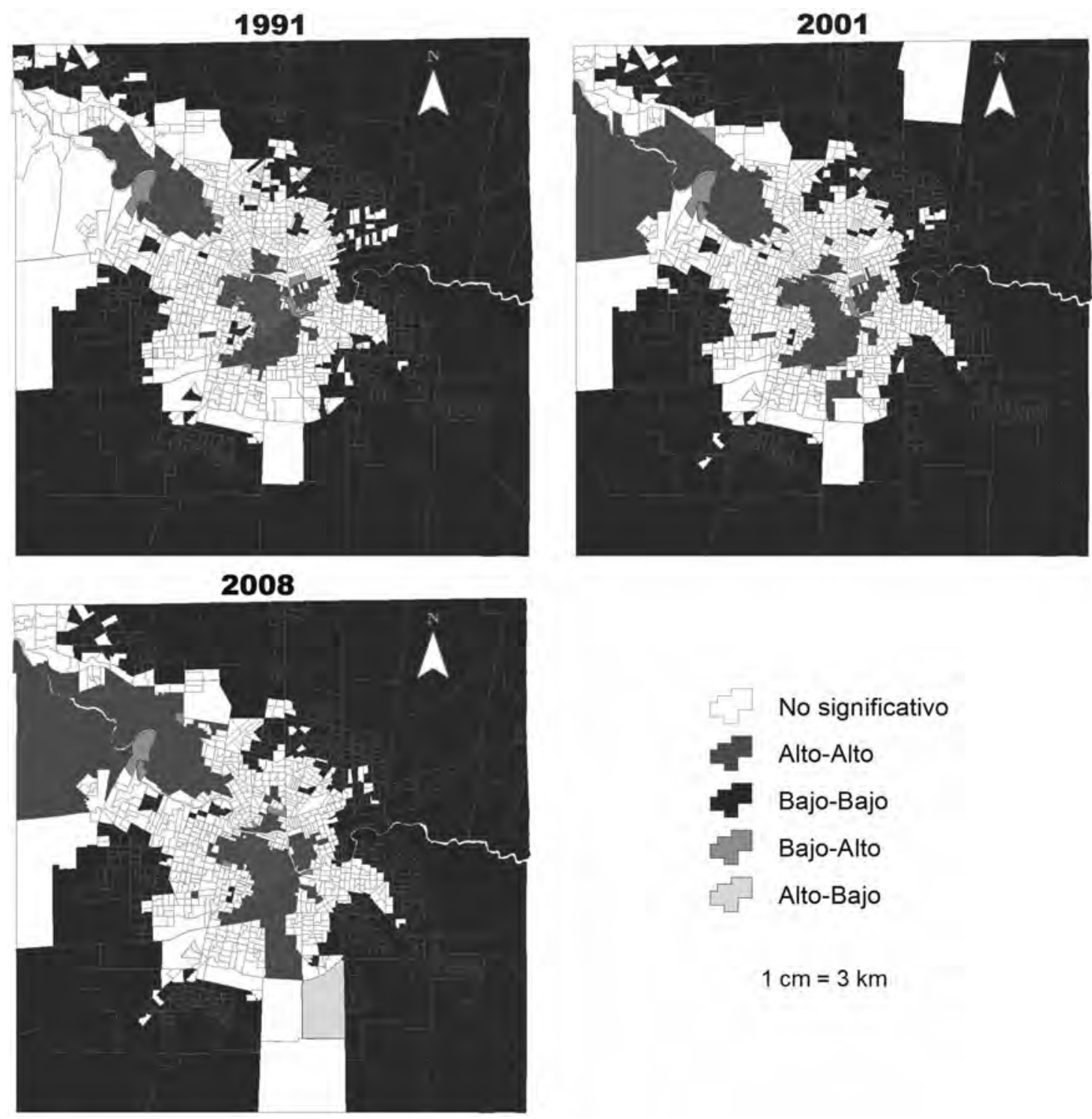

$1 \mathrm{~cm}=3 \mathrm{~km}$

Fuente: elaboración propia con base en información de los Censos 1991, 2001 y 2008 y cartografía oficial de INDEC. 


\section{LOCALIZACIÓN EN EL ESPACIO URBANO DE LAS ÁREAS DE SEGREGACIÓN ${ }^{48}$}

Los jefes de hogar con educación baja presentan un patrón de autocorrelación espacial Alto-Alto en el área periférica de Norte, Sur y Este de la ciudad, lo cual señala que allí se encuentran radios con alto porcentaje (superior a la media local) de jefes poco educados rodeados de otros radios con la misma característica. Asimismo tienen un patrón de autocorrelación espacial Bajo-Bajo en los radios localizados en zonas centrales y pericentrales de la ciudad. A diferencia del otro patrón, los radios identificados con patrón Bajo-Bajo indican que son zonas con bajo porcentaje (inferior a la media local) de jefes con educación baja circundados de otras con similares atributos (Figura 2).

Por su parte, los jefes de hogar con educación media-baja exhiben un patrón de autocorrelación espacial similar al observado para el grupo anterior; aunque se destaca al final de período una consolidación de dicho patrón en la periferia Norte, Sur y Este de la ciudad. En contraste, se observa una baja concentración de este grupo en la zona central y en el corredor Noroeste (Figura 3).

48 Considerando la estabilidad de los valores del I de Moran Global ante cambios en el tipo de contigüidad utilizada, en el análisis local sólo se trabajó a partir del críterio Queen, que considera como vecinos a todos los radios que comparten cualquier frontera común.
El patrón de autocorrelación espacial se invierte cuando se analiza la distribución espacial de los jefes con educación media-alta, ya que las zonas con alta concentración de jefes en esta condición rodeadas de otras similares se localizan en el área central de la ciudad y las zonas identificadas como Bajo-Bajo en el área Sur, Sureste, Este y Noreste. Además, este patrón se mantiene casi inalterado durante el período analizado (Figura 4).

Los jefes de hogar con mayor nivel educativo presentan un patrón de localización espacial similar al observado para el grupo anterior, excepto que las zonas con autocorrelación Alta-Alta y Baja-Baja se extienden, hacia el corredor Noroeste y el Noreste, respectivamente (Figura 5).

A partir del análisis de la autocorrelación espacial de los jefes de hogar según su nivel educativo, puede deducirse, y confirmar lo observado a partir de los indicadores de concentración espacial, que la ciudad de Córdoba se encuentra altamente segregada en términos socioeconómicos. Es decir, que los hogares, según el nivel educativo de sus jefes, tienden a localizarse en forma no aleatoria en el espacio geográfico considerado, erigiendo vastas 
áreas homogéneas que permanecen casi inalteradas en el período analizado. Por un lado, los jefes con educación baja y media-baja tienden a concentrarse en el Sur, el Este y el Norte de la ciudad, conformando áreas con "segregación por pobreza". Por otro lado, los jefes con educación media-alta y alta ocupan predominantemente la zona central y el corredor Noroeste, constituyendo zonas caracterizadas por una "segregación por riqueza". Aunque, para el caso particular de los jefes con educación alta, también se observa la presencia de concentración espacial hacia la zona Centro-Sur de la ciudad, especialmente en 2001 y 2008.

\section{Conclusiones y reflexiones}

Los resultados obtenidos en este trabajo sugieren que la ciudad de Córdoba presenta significativos niveles de segregación residencial socioeconómica, aproximada a partir de la distribución geográfica de los jefes de hogar según su máximo nivel educativo alcanzado, y que los mismos no experimentaron retrocesos entre 1991 y 2008, sino que, muy por el contrario, vieron estancados o, en algunos grupos, incrementados sus valores. Ello permite afirmar no solo que los diferentes grupos sociales muestran un patrón residencial bien definido, ocupando y apropiándose de zonas específicas del espacio urbano, sino que el mismo se ha visto reafirmado en las últimas décadas, a pesar de las mejoras observadas en términos educativos.

A pesar de las dificultades inherentes a la comparación de estos resultados con los encontrados en otras ciudades latinoamericanas, debido principalmente a la diversidad de atributos socioeconómicos, índices y escalas de desagregación utilizadas, se evidencia que la ciudad de Córdoba exhibe, para las rondas censales de 1990 y 2000, niveles de segregación -medidos a partir de índices de concentración espacial- superiores a los registrados en esas ciudades, independientemente de sus tamaños poblacionales ${ }^{49}$. Del grupo de investigaciones comparables, la ciudad de Córdoba sólo presenta valores inferiores, aunque cercanos, a los obtenidos por Torres ${ }^{50}$ para la mega-ciudad de São Paulo entre los grupos ubicados en los extremos de la escala educativa.

Respecto a la segregación en la primera década del siglo XXI, se destaca que no pueden realizarse comparaciones por no hallarse trabajos que analicen dicho fenómeno en ese período, posiblemente porque aún no se encuentran disponibles los microdatos de la ronda censal de 2010 en gran parte de los países latinoamericanos.

49 Gómez, 2011; Rodríguez, 2008; Peláez, González y Cunha, 2008; Prieto, Formiga y Medus, 2011; Roberts y Wilson, 2009.

50 Torres, 2004. 
Las evidencias encontradas en este trabajo sugieren la presencia de una segregación residencial que se manifiesta en una ocupación diferencial del espacio urbano, pero no en una alta homogeneidad social de las áreas residenciales, ya que el indicador de aislamiento exhibió valores relativamente bajos. Cabe destacar que estos valores posiblemente sean producto de la artificialidad de las unidades de desagregación en las que los datos son presentados y la segregación residencial medida, las cuales son definidas para facilitar el relevamiento censal y que no suponen relevancia en términos socioeconómicos.

Sin embargo, similar a lo observado en otras ciudades $^{51}$, se destaca que los hogares ubicados en extremo superior de la escala social son los que se encuentran más aislados; lo cual, sumado a su alta concentración espacial, indicaría que el patrón residencial de este grupo se acercaría, en terminología weberiana, a un enclaustramiento excluyente. Esto último tendría su expresión física, tal como lo resalta Marengo ${ }^{52}$, en el progresivo número de urbanizaciones cerradas exclusivas que se han desarrollado desde finales de 1980 en la periferia Noroeste y desde comienzos del siglo XXI en la zona Sur de la ciudad. Este rápido incremento se da paralelamente a los cambios en la provisión

51 Gómez, 2011; Rodríguez, 2008; Groisman y Suárez, 2006; PNUD, 2009; Roberts y Wilson, 2009.

52 Marengo, 2002, 2004. de servicios e infraestructuras para consumo y recreación (shopping centers, supermercados, hipermercados, complejos de salas de cine, entre otros), que también se localizan en las periferias de la ciudad. Roberts y Wilson ${ }^{53}$ sugieren que estos cambios en los patrones residenciales y comerciales son propiciados por la creciente expansión de la infraestructura de transporte que hace a las localizaciones periféricas más atractivas para las clases media-altas y altas, como así también en la diversidad de la oferta inmobiliaria para estos sectores. Asimismo, estos autores ${ }^{54}$ advierten que la fragmentación y la relativa baja escala de las urbanizaciones cerradas no posibilitan hablar de un suburbanización de las elites, tal como se observa en las ciudades norteamericanas.

En forma simultánea, se registra un aumento en la concentración espacial y, en menor medida, en el aislamiento social de los hogares con jefes con menores niveles educativos, lo cual podría tener su origen en las diferentes políticas públicas habitacionales ejecutadas en las últimas décadas y particularmente, a las relocalizaciones de asentamientos informales a grandes complejos habitacionales construidos en la periferia pobre y semi-rural de la ciudad por el Gobierno de la Provincia de Córdoba entre 2003 y 2007. Diversos estudios ${ }^{55}$ dan cuenta

53 Roberts y Wilson, 2009.

54 lbíd.

55 Elorza, 2009; PNUD, 2009; Tecco y Valdés, 2006, entre otros. 
de los posibles efectos e impactos negativos que esta concentración espacial de hogares pobres podrían tener sobre la vida cotidiana de las familias trasladas, en especial en lo referido a la reproducción generacional de la pobreza.

Los cambios registrados en los patrones residenciales de los grupos sociales ubicados en los extremos de la escala educativa dan cuenta de la consolidación del grado de segregación residencial existente entre estos dos grupos: por ejemplo, hacia 2008 casi el 70\% de los jefes con educación alta (o baja) deberían cambiar su lugar de residencia (radio censal) para obtener una distribución homogénea en toda la ciudad. A pesar que estos valores son similares a los observados entre negros y blancos en las áreas metropolitanas más segregadas de los Estados Unidos en $2000^{56}$, tal comparación debe ser tomada con muchas reservas, principalmente por las diferencias en el tamaño de escalas utilizadas (los sectores censales -census tract- norteamericanos son una unidad espacial intermedia entre los radios y las fracciones censales aquí considerados) y por la dimensión de la segregación medida (racial en los Estados Unidos y socioeconómica en la Argentina).

Pero, ¿cómo se expresa en el espacio geográfico de la ciudad dichos niveles de segregación cuando se analizan las áreas residenciales respecto a las vecinas? Los

56 CensusScope, sin año. jefes de hogar, según su máximo nivel educativo alcanzado, exhiben en patrón de distribución no aleatorio y altamente concentrado en el espacio urbano, independientemente del criterio de vecindad utilizado. Ello significa que no sólo los grupos se concentran en específicas zonas del espacio residencial según su nivel educativo, sino que también la composición social de las áreas internas de la ciudad se encuentra fuertemente asociada a la de sus vecinos, conformado formas positivas de autocorrelación espacial. Semejantes resultados fueron hallados en un estudio comparativo para siete ciudades latinoamericanas compilado por Roberts y Wilson ${ }^{57}$, en las cuales también se observan altos niveles de clustering de áreas censales con características similares.

Más allá de la presencia de esta estructura global de dependencia espacial, ise visualizan patrones de aglomeración y/o localizaciones atípicas locales? Mientras en la periferia Norte-Este-Sur de la ciudad predominan áreas residenciales con segregación por pobreza, la zona central y el corredor Noroeste se caracterizan por un patrón residencial representado por una segregación por riqueza. En las zonas semi-periféricas de la ciudad se encuentran las denominadas áreas heterogéneas que, en su conjunto, actúan como una especie de franja de amortiguación entre los anteriores espacios homogéneos. En menor medida, se observan localizaciones atípicas, 
es decir, espacios que no comparten la composición social de sus vecinos.

Respecto a las variaciones registradas, se observa que en 2008 la periferia de la ciudad se caracterizaba por seguir albergando hogares cuyos jefes tienen bajo nivel educativo, manteniéndose la ocupación diferencial del espacio observada a principios de la década de 1990. Esto último posiblemente se encuentra asociado, como se mencionara anteriormente, a la relocalización de casi 7.000 familias que residían en asentamientos informales a complejos habitacionales construidos en zonas anteriormente pobres, reforzando la concentración espacial de la pobreza ya existente.

Los hogares con jefes con educación secundaria/ media completa o más mantienen su localización predominante en el área central, las zonas pericentrales y el corredor Noroeste de la ciudad. Aunque, hacia principios del siglo XXI, los hogares pertenecientes al extremo superior de la escala educativa comienzan a abandonar sus tradicionales zonas de residencia, corredor Noroeste de la ciudad, y a ocupar zonas periféricas anteriormente caracterizadas como pobres o heterogéneas. Este cambio en el patrón residencial puede explicarse, como se comentara anteriormente, por la adopción del diseño de barrio cerrado (countries) como estrategia de expansión de la oferta inmobiliaria para los sectores más pudientes de la sociedad. Este diseño, sumado a la significativa superficie de los proyectos, posibilitó el reforzamiento de las distancias sociales entre estos sectores y aquellos que residían con anterioridad en esas zonas.

En síntesis, el presente no sólo permite realizar una aproximación objetiva al fenómeno de segregación residencial en la ciudad de Córdoba y conocer cómo este ha evolucionado desde comienzos de la década de 1990 hacia finales de la primera década del siglo XXI, sino que también los resultados aquí alcanzados, en especial los referidos a la identificación de las áreas residenciales, representan una herramienta indispensable para el diagnóstico, diseño, ejecución y evaluación de cualquier política pública que tenga por objetivo alcanzar una mayor interacción física entre personas de distintos grupos sociales, un mayor acceso a la ciudad por parte de los pobres y el debilitamiento de los estigmas territoriales.

Finalmente, cabe destacar que el estudio de la segregación residencial, cualquiera sea su dimensión, no concluye de ninguna manera en el diagnóstico objetivo de su situación actual y evolución. Por el contrario, dicho diagnóstico actúa como disparador de nuevas líneas de investigación orientadas a profundizar qué causas o determinantes permiten explicar la conformación y evolución de los patrones residenciales de segregación existentes, algunos de ellos explorados en este trabajo, y/o explorar qué efectos o impactos puede tener la segregación residencial sobre las condiciones de vida de 
los residentes de áreas segregadas, especialmente de aquellos más pobres.

\section{Bibliografía}

ALVES, Fátima, FRANCO, Creso y RIBEIR0, Luiz César de Queiroz. Segregación urbana y rezago escolar en Río de Janeiro [En línea]. Revista de la CEPAL. (94): 133-148, abril 2008. [Fecha de consulta: 14 de mayo de 2012]. ISSN 1682-0908. Disponible en: http://www.eclac.cl/publicaciones/xml/8/327 28/94eAlvesFrancodeQueiroz.pdf

ANSELIN, Luc. Local indicators of spatial association - LISA. [En línea] Geographical Analysis. 27(2): 93 115, April 1995. [Fecha de consulta: 25 de junio de 2010]. ISSN 1538-4632. Disponible en: http:// dx.doi.org/10.1111/j.1538-4632.1995.tb00338.x

ASSUNÇA0, Renato M. y REIS, Edna A. A new proposal to adjust Moran's / for population density. [En línea]. Statistics in medicine. 18(16): 2147-2162, August 1999. [Fecha de consulta: 25 de noviembre de 2012]. ISSN 1097-0258. Disponible en: http://dx.doi.org/10.1002/ (SICI)1097-0258(19990830)18:16<2147::AIDSIMl79>3.0.CO;2-I

BOURDIEU, Pierre. Efectos de lugar. En: BOURDIEU, Pierre (Ed.). La miseria del mundo. Madrid, España, Fondo de Cultura Económica. 1999. p. 119124. ISBN 95-0-557270-0.

BRUN, Jacques. Essai critique sur la notion de ségrégation et sur son usage en géographie urbaine. En:
BRUN, Jacques, ed. y RHEIN, Catherine, ed. La ségrégation dans la ville. Concepts et mesures. Paris, France, L'Harmattan. 1994. p. 21-57. ISBN 978-2-73842-477-8.

BRUN, Jacques y RHEIN, Catherine (Eds.). La ségrégation dans la ville. Concepts et mesures. Paris, Francia, L'Harmattan, 1994. 258 p. ISBN 978-2-73842-477-8.

CALDEIRA, Teresa Pires do. Ciudad de muros. Barcelona, España, Gedisa. 2007. 479 p. ISBN 978-8-49784-191-7.

CAPRON, Guénola y GONZÁLEZ ARELLANO, Salomón. Las escalas de la segregación y de la fragmentación urbana. Trace. (49): 65-75, junio 2006. [Fecha de consulta: 20 de mayo de 2011]. ISSN 0185-626.

CENSUSSCOPE (United States). Segregation: Dissimilarity Indices US Metro Areas Ranked by White/ Black Dissimilarity Index. [En línea]. CensusScope. Sin año. Disponible en: http://www.censusscope.org/us/rank_dissimilarity_white_black. html

CUNHA, José Marcos Pinto da y JIMÉNEZ, Maren Andrea. The Process of Cumulative Disadvantage: Concentration of Poverty and the Quality of Public Education in the Metropolitan Region of Campinas. En: ROBERTS, Bryan R., ed. y WILSON, Robert H., ed. Urban Segregation and Governance in the Americas. New York, United States of America, Palgrave Macmillan. 2009. p. 169-185. ISBN 0-230-60960-0. 
CUNHA, José Marcos Pinto da y JAKOB, Alberto Augusto Eichman. Segregação socioespacial e inserção no mercado de trabalho na Região Metropolitana de Campinas. [En línea]. Revista Brasileira de Estudos de População. 27(1): 115-139, jan. 2010. Disponible en: http://dx.doi.org/10.1590/ S0102-30982010000100008

ELORZA, Ana Laura. Política de erradicación de Villas: impactos en la calidad de vida de las familias relocalizadas. Estudio de caso: Ciudad de los Niños. [En línea]. Dossier de Confluencias. 15(67): 1-23, diciembre 2009. [Fecha de consulta: 21 de junio de 2011]. Disponible en: http://www.cpsscba.org/ Revista/Digital67/dossier-67.pdf

GÓMEZ, Néstor J. Segregación residencial en el Gran Santa Fe a comienzos del siglo XXI [En línea]. Bitácora urbano/territorial. 19(2): 63-74, julio 2011. [Fecha de consulta: 26 de septiembre de 2012]. ISSN 2027-145X. Disponible en: http://www.redalyc.org/articulo.oa?id=74822445006

GRAFMEYER, Yves. Regards sociologiques sur la ségrégation. En: BRUN, Jacques, ed. y RHEIN, Catherine, ed. La ségrégation dans la ville. Concepts et mesures. Paris, Francia, L'Harmattan, 1994. p. 85-117. ISBN 978-2-73842-477-8.

GROISMAN, Fernando y SUÁREZ, Ana Lourdes. Segregación residencial en la Ciudad de Buenos Aires. [En línea]. Población de Buenos Aires. 3(4): 27-37, octubre 2006. [Fecha de consulta: 12 de octubre de 2010]. ISSN 1668-5458. Disponible en: http://www.redalyc.org/articulo. oa? id $=74030403$

ARTíCULO: Segregación residencial socioeconómica en la ciudad de Córdoba (Argentina) Tendencias y patrones espaciales / Florencia Molinatti
KAZTMAN, Rubén y RETAMOSO, Alejandro. Segregación espacial, empleo y pobreza en Montevideo. [En línea]. Revista de la CEPAL. (85): 131-148, abril 2005. [Fecha de consulta: 9 de junio de 2009]. ISSN 1682-0908. Disponible en: http://www. eclac.org/publicaciones/xml/8/21048/lcg2266eKaztmanRetamoso.pdf

- Efectos de la segregación urbana sobre la educación en Montevideo. [En línea] Revista de la CEPAL. (91): 133-152, abril 2007. [Fecha de consulta: 9 de junio de 2009]. ISSN 1682-0908. Disponible en: http://www.eclac.cl/publicaciones/xml/3/28263/ LCG2333eKaztman.pdf

LATTES, Alfredo E. Población urbana y urbanización en América Latina. En: CARRIÓN, F., ed. La ciudad construida. Urbanismo en América Latina. Quito, Ecuador, FLACSO. 2001. p. 49-76. ISBN 9978-67-057-2.

LINARES, Santiago. Análisis y modelización de la segregación socioespacial en ciudades medias bonaerenses mediante Sistemas de Información Geográfica: Pergamino, Olavarría y Tandil (19912001) [En línea]. Revista Geográfica de Valparaíso. (45): 3-22, 2012. [Fecha de consulta: 14 de agosto de 2012]. ISSN 0718-9877. Disponible en: http:// www.rgv.ucv.cl/Articulo_1.pdf

MARCUSE, Peter. Enclaves yes, ghettoes, no: segregation and the State. [En línea]. The Centre for Urban and Community Studies. July 2001. [Fecha de consulta: 13 de mayo de 2011]. Disponible en: http:// www.urbancentre.utoronto.ca/pdfs/curp/Marcuse_Segregationandthe.pdf

revista invi ํำ 79 / Noviembre 2013 / Volumen N ${ }^{\circ} 28: 61-94$ 
MARENG0, Cecilia. Una aproximación a la segregación residencial, como punto de partida en la formulación de políticas. [En línea]. Boletín del Instituto de la Vivienda. 19(50): 167-183, mayo 2004. [Fecha de consulta: 25 de mayo de 2012]. ISSN 07165668. Disponible en: http://www.revistas.uchile. cl/index.php/INVI/article/viewFile/8813/8620

- Estrategias habitacionales en los bordes urbanos. Puntos de partida para analizar la expansión suburbana. [En línea]. Proyección. 2(1), 2002. [Fecha de consulta: 16 de marzo de 2011]. ISSN 16670876. Disponible en: http://bdigital.uncu.edu. ar/3112

MASSEY, Douglas S. y DENTON, Nancy A. The Dimensions of Residential Segregation. [En línea]. Social Forces. 67(2): 281-315, December 1988. [Fecha de consulta: 28 de febrero de 2010]. ISSN 1534-7605. Disponible en: http://dx.doi.org/10.1093/sf/67.2.281

MOLINATTI, Florencia. Segregación residencial e inserción laboral en la ciudad de Córdoba. [En línea]. EURE. 39(117): 117-145, mayo 2013. [Fecha de consulta: 07 de mayo de 2013]. ISSN 07176235. Disponible en: http://dx.doi.org/10.4067/ S0250-71612013000200006

MOLINATTI, Florencia y PELÁEZ, Enrique. Los patrones espaciales de los comportamientos de riesgo en la ciudad de Córdoba (Argentina) 2001. [En línea]. Revista Brasileira de Estudos de População. 29(1): 37-52, janeiro 2012. [Fecha de consulta: 18 de julio de 2012]. ISSN $0102-$ 3098. Disponible en: http://dx.doi.org/10.1590/ S0102-30982012000100003
MUNICIPALIDAD de Córdoba (Argentina). La ciudad de 1984 y la ciudad de 2008. Transformaciones en la ciudad desde 1984, fecha del último revalúo general [En línea]. Municipalidad de Córdoba. 2008. Disponible en: http://www.cordoba.gov.ar/cordobaciudad/principal2/docs\%5Ccatastro\%5Cla\%20 ciudad\%20de\%201984\%20y\%202008c.pdf

PELÁEZ, Enrique, GONZÁLEZ, Leandro y CUNHA, José Marcos Pinto da. Dimensiones de la segregación residencial en el Gran Córdoba (Argentina) y comparación con Región Metropolitana de Campinas (Brasil) [En línea]. Carta Económica Regional. 20(100): 31-47, septiembre 2008. [Fecha de consulta: 05 de febrero de 2012] ISSN 0187-7674. Disponible en: http://cartaeconomica.cucea.udg. mx/administracion/uploads/articulo825.pdf

PRIETO, María Belén, FORMIGA, Nidia y MEDUS, Silvina. La segregación socio-residencial en ciudades intermedias. El caso de Bahía Blanca-Argentina. [En línea]. XIII Conferencia Iberoamericana en Sistemas de Información Geográfica (CONFIBSIG). 2011. [Fecha de consulta: 15 de febrero de 2013] Disponible en: http://www.inegi.org.mx/eventos/2011/conf_ibero/doc/ET4_19_PRIETO.pdf

PNUD Programa de Naciones Unidas para el DesarroIlo. Aportes para el desarrollo humano en Argentina 2009. Segregación residencial en Argentina [En línea]. Buenos Aires, Argentina, Programa de Naciones Unidas para el Desarrollo. 2009. 51 p. [Fecha de consulta: 20 de septiembre de 2010]. Disponible en: http://www.undp.org.ar/docs/Libros_y_Publicaciones/PNUD_segregacion_novl7-screen.pdf 
REARDON, Sean F. y O'SULLIVAN, David. Measures of spatial segregation. [En línea] Sociological Methodology. 34(1): 121-162, 2004. [Fecha de consulta: 26 de febrero de 2010]. ISSN 1467-9531. Disponible en: http://dx.doi. org/10.1111/j.0081-1750.2004.00150.x

REBORD, Gustavo. Irregularidad en la tenencia de la propiedad. [En línea]. HOY la Universidad. 2 (3): 42-47, junio 2010. [Fecha de consulta: 26 de noviembre de 2010]. ISSN 1667-6289. Disponible en: http://www.unc.edu.ar/seccion/divulgacion/ hoylauniversidad/numero3/

ROBERTS, Bryan R., ed. y WILSON, Robert H., ed. Urban segregation and governance in the Americas. New York, United States of America, Palgrave Macmillan. 2009. 231 p. ISBN 0-230-60960-0.

RODRÍGUEZ, Gonzalo. Segregación residencial socioeconómica en la Ciudad Autónoma de Buenos Aires. Dimensiones y cambios entre 1991-2001 [En línea]. Población de Buenos Aires. 5(8): 7-30, octubre 2008. [Fecha de consulta: 18 de mayo de 2010]. ISSN 1668-5458. Disponible en: http://www.redalyc.org/articulo. oa?id $=74011761001$

RODRíGUEZ, Jorge y ARRIAGADA, Camilo. Segregación residencial en la ciudad latinoamericana. [En línea]. EURE. 30(89): 5-24, mayo 2004. [Fecha de consulta: 12 de mayo de 2009]. ISSN 07176236. Disponible en: http://dx.doi.org/10.4067/ S0250-71612004008900001

ARTíCULO: Segregación residencial socioeconómica en la ciudad de Córdoba (Argentina): Tendencias y patrones espaciales / Florencia Molinatti
RODRÍGUEZ VIGNOLI, Jorge. Segregación residencial socioeconómica: ¿qué es?, ¿cómo se mide?, ¿qué está pasado?, ¿importa? Santiago de Chile, Chile, Centro Latinoamericano y Caribeño de Demografía (CELADE), Naciones Unidas. 2001. 80 p. Serie Población y Desarrollo $N^{\circ} 16$. ISBN 92-1-321882-6.

SABATINI, Francisco. La segregación social del espacio en las ciudades de América Latina. Santiago de Chile, Chile, Instituto de Estudios Urbanos y Territoriales / Pontificia Universidad Católica de Chile, 2003. 64 p. Serie Azul No 35.

SABATINI, Francisco, CÁCERES, Gonzalo y CERDA, Jorge. Segregación residencial en las principales ciudades chilenas: Tendencias de las últimas tres décadas y posibles cursos de acción. [En línea]. EURE. 27(82): 21-42, diciembre 2001. [Fecha de consulta: 3 de marzo de 2011]. ISSN 07176236. Disponible en: http://dx.doi.org/10.4067/ S0250-71612001008200002

SABATINI, Francisco; WORMALD, Guillermo; SIERRALTA, Carlos y PETERS, Paul A. Residential Segregation in Santiago: Scale-Related Effects and Trends, 1992-2002. En: ROBERTS, Bryan R., ed. y WILSON, Robert H., ed. Urban Segregation and Governance in the Americas. New York, United States of America, Palgrave Macmillan. 2009. p. 121-143. ISBN 0-230-60960-0.

SALAS Vanegas, Andrea. Ségrégation résidentielle et production du logement à Bogotá, entre images et réalités (Segregación residencial y producción de

revista invi ํำ / Noviembre 2013 / Volumen N² 28: 61-94 
vivienda en Bogotá, entre imágenes y realidades). [En línea]. Poitiers, France, Université de Poitiers, 2008. 455 p. [Fecha de consulta: 25 de agosto de 2010]. Disponible en: http://tel.archives-ouvertes. fr/tel-00303317/

SVAMPA, Maristella. La brecha urbana. Countries y barrios privados en Argentina. Buenos Aires, Argentina, Capital Intelectual. 2004. 111 p. ISBN: 987-1181-10-8.

TECCO, Claudio y VALDÉS, Estela. Segregación residencial socioeconómica (SRS) e intervenciones para contrarrestar sus efectos negativos. Reflexiones a partir de un estudio en la ciudad de Córdoba, Argentina. [En línea]. Cuadernos de Geografía. (15): 53-66, 2006. [Fecha de consulta: 23 de marzo de 2011]. ISSN 0121-215X. Disponible en: http:// www.redalyc.org/articulo.oa?id=281821952005
TORRES, Horacio da Gama. Segregação residenciale políticas públicas: São Paulo na década de 1990. [En línea]. Revista Brasileira de Ciências Sociais. 19(54): 41-55, 2004. [Fecha de consulta: 16 de junio de 2009]. ISSN 0102-6909. Disponible en: http:// dx.doi.org/10.1590/S0102-69092004000100003

VAPÑARSKY, César A. Primacía y macrocefalia en la Argentina: la transformación del sistema de asentamiento humano desde 1950. Desarrollo Económico. 35(138): 227-254, julio 1995. ISSN 0046-001X.

WHITE, Michael. The measurement of spatial segregation. [En línea]. The American Journal of Sociology. 88(5): 1008-1018, March 1983. [Fecha de consulta: 26 de febrero de 2011]. ISSN ISSN 0002-9602. Disponible en: http://www.jstor.org/ stable/2779449 